\title{
Probing Functionality for Energy Related Materials; Opportunities for Advanced Electron Microscopy
}

\author{
Peter A. Crozier ${ }^{1}$ \\ 1. School for the Engineering of Matter, Transport and Energy, Arizona State University, Tempe Arizona \\ 85287-6106.
}

The demand for developing new energy technologies continues to push characterization tools, including those based on electrons, x-rays and neutrons, to tackle the high level of complexity found in active materials for energy conversion processes. As pointed out in the recent DOE report, the frontiers of basic energy research require new generations of instrumentation to understand complex materials and chemical systems, energy systems in realistic working environments, and systems that are dynamic, far from equilibrium, and extremely heterogeneous [1]. Electrons, x-rays and neutrons offer unprecedented opportunities to interrogate structure and charge transfer functionalities in energy materials based on thermochemical, photochemical and electrochemical processes. To understand functionality, it is important to characterize these systems under real world conditions i.e. in situ and operando. Another key objective is developing a fundamental relationship between structure, composition, bonding and functionality across a wide range of different length scales. Electron, x-ray and neutron scattering approaches can all provide critical pieces of information which can be brought together to give a more complete picture. Multi-modal approaches, in which combinations of techniques can be applied to the same sample, are an aspirational goal which has only been realized in a very limited number of cases. Charge transfer and chemical processes may occur on time scales as short as a few femtoseconds, necessitating the development of ultrafast approaches.

We have developed and employed (scanning) transmission electron microscopy ((S)TEM) imaging and spectroscopy to deliver subnanometer views of structural features which cannot be adequately interrogated with broad beam x-ray or neutron techniques. More specifically, the ability to probe and understand nanoparticle surfaces and shapes, interfaces, grain boundaries, fine scale structural, compositional and bonding heterogeneity is critical for understanding charge transfer processes. The enormous advances in electron optics has pushed spatial resolutions below $1 \AA$ for imaging and $1-2 \AA$ for spectroscopy. Electron energy-loss spectroscopy (EELS) has also undergone a revolution with $10 \mathrm{meV}$ energy resolution now possible. We have been able to use this approach to investigate electronic, optical and vibrational properties of nanoscale systems [2-9]. STEM bright and dark-field images along with techniques like negative $\mathrm{C}_{\mathrm{s}}$ imaging now allow atomic columns to be observed with atomic number spanning the entire periodic table in favorable cases.

The power of TEM is its ability to provide atomic-level information on small volumes of material. This is often adequate for well-defined epitaxial systems, but for more complex heterogeneous materials systems found in many energy conversion technologies, it is difficult to make a statistical connection to larger length scale functionalities like electrical conductivity and catalysis. Multi-modal approaches are emerging to provide information that spans multiple length scales. For example, in polycrystalline solid electrolytes of relevance to fuels cells and batteries, grain boundaries often play an important role in regulating ionic transport. By combining electron backscatter diffraction in a scanning electron microscope with STEM precession electron diffraction and EELS, it becomes possible to infer the nanoscopic characteristic of 100,000 grain boundaries and relate this to macroscopic grain boundary 
conductivity $[5,10]$. The continued development of diverse techniques and more sophisticated data sampling will allow a stronger statistical link between atomic-level structure, composition and bonding with macroscopic functionality.

In order to relate materials structure to functionality for technological applications, it is important to characterize materials under real conditions. By real conditions we mean that the material is subject to stimuli such as heat, light, electric fields, gas or liquid environments which may trigger changes in the functionally relevant bulk, surface or interface structures. These changes may be in the form of gross phase transformations or subtler dynamic changes in either structure or bonding. In situ characterization tools based on electrons, x-rays or neutrons may capture these changes and allow the material forms to be determined under working conditions. For example, we have recently employed in situ TEM to investigate the oxygen exchange and carbon deposition process on the surfaces of metal nanoparticles supported on reducible oxides [11]. However, not all changes that occur in a material under in situ conditions are related to functionality. One area where this is often true is in the field of heterogeneous catalysis where many surface structures may be present under reaction conditions, but most may not be associated with the active site performing catalysis. To relate structure to functionality it may be necessary to measure functionality simultaneously with structure leading to so-called operando approaches $[12,13]$. The functionality may be catalysis, electronic or ionic current, materials growth, photon emission/absorption, etc..., and it is usually associated with some form of charge transfer process. The technologically relevant functionality is often a macroscopic collective effect which is statistical in nature and it may be challenging to identify structurally relevant motifs. One approach to address this limitation is to vary the conditions to cause dynamic changes in structure and functionality and then correlate the observations [14]. The ability to succeed with such an approach will depend on the number of structural variants observed under operando conditions. In many cases, it is necessary to employ modelling approaches such as density functional theory to correlate structural motifs with charge transfer and functionally relevant electronic structure. It is clear that with the continued rate of developments in instrumentation, TEM will continue to provide transformative information on energy materials.

References:

[1] US Department of Energy Report, Basic Research Needs for Innovation and Discovery of Transformative Experimental Tools. (2017)

[2] K.Venkatraman et al., Microscopy (2018) 1-10

[3] Q. Liu et al, Ultramicroscopy, 178 (2017) p. 2-11.

[4] P.A.Crozier, P.A., Ultramicroscopy, 180 (2017) p. 104-114.

[5] W.J. Bowman et al., Nanoscale, 9 (2017) p. 17293 - 17302.

[6] P.A.Crozier et al, Ultramicroscopy, 169 (2016) p. 30-36.

[7] W.J. Bowman et al., Ultramicroscopy 167 (2016) p. 5-10.

[8] O.L. Krivanek et al., Nature, 514 (2014) p. 209-212.

[9] Q. Liu et al., (submitted) 2018.

[10] W.J. Bowman et al (submitted), 2018.

[11] E.L. Lawrence, and P.A. Crozier, (submitted) 2018.

[12] F. Tao, and P.A. Crozier, Chemical Reviews, 116(6) (2016) p. 3487-3539.

[13] S. Chenna, and P.A. Crozier, ACS Catalysis, 2 (2012) p. 2395-2402.

[14] B.K. Miller and P.A. Crozier, (in preparation), 2018.

[15] We gratefully acknowledge support from NSF DMR-1308085, NSF CBET-1604971, NSF CHE1508667 and DOE (DE-SC0004954). 